Revue d'histoire de l'Amérique française

REVUE D.HISTOIRE DE L'AMÉRIQUE FRANÇAISE

\title{
Aperçu critique des principaux ouvrages pouvant servir à l'histoire du développement minier de l'Abitibi-Témiscamingue (1910-1950)
}

\section{Benoît-Beaudry Gourd}

Volume 30, numéro 1, juin 1976

URI : https://id.erudit.org/iderudit/303512ar

DOI : https://doi.org/10.7202/303512ar

Aller au sommaire du numéro

Éditeur(s)

Institut d'histoire de l'Amérique française

ISSN

0035-2357 (imprimé)

1492-1383 (numérique)

Découvrir la revue

Citer cette note

Gourd, B.-B. (1976). Aperçu critique des principaux ouvrages pouvant servir à l'histoire du développement minier de l'Abitibi-Témiscamingue (1910-1950).

Revue d'histoire de l'Amérique française, 30(1), 99-107.

https://doi.org/10.7202/303512ar d'utilisation que vous pouvez consulter en ligne. 
NOTE CRITIQUE

\title{
APERÇU CRITIQUE DES PRINCIPAUX OUVRAGES POUVANT SERVIR À \\ L'HISTOIRE DU DÉVELOPPEMENT MINIER DE L'ABITIBI-TÉMISCAMINGUE (1910-1950)
}

\author{
BENOÎT-BEAUDRY GOURD \\ Noranda
}

L'Abitibi-Témiscamingue constitue une région un peu à part dans le développement du Québec. C'est un au-delà isolé de l'axe laurentien dont le peuplement est relativement récent. Le Témiscamingue ne se peuple véritablement qu'à partir de 1885 et les premiers établissements permanents en Abitibi remontent à 1912. La population de la région passe de 6,685 habitants en 1901 à 141,458 en 1951. La croissance démographique apparaît particulièrement marquée durant les décennies de 1911-1921 et de 1931-1941 avec des taux d'augmentation de $156.5 \%$ et de $144.1 \%{ }^{1}$. Le développement de l'Abitibi-Témiscamingue va donner vie à trois sous-régions possédant chacune au départ certaines particularités socio-économiques qui s'estomperont lentement avec le temps: le vieux Témiscamingue agricole, l'Abitibi rural et la zone minière de la Faille de Cadillac.

Il n'existe encore à ce jour aucune véritable synthèse historique décrivant et expliquant l'évolution de l'Abitibi-Témiscamingue. De plus les recherches historiques ont surtout porté sur la colonisation agricole. Cela tient peut-être au fait que, contrairement à la colonisation agricole qui est tout entière l'œuvre du Canada français, le développement minier de la région découle du débordement de l'industrie minière nord-ontarienne dans le Québec voisin. Il n'est pas étonnant alors qu'on sê soit avant tout préoccupé de mettre en

1 Fédération des Chambres de commerce de l'Abitibi, Commissariat industriel de l'Abitibi, Quelques perspectives économiques de la région abitibienne (Senneterre, 1956), 45.

RHAF, vol. 30, no 1 (juin 1976) 
relief les réalisations de la collectivité québécoise. Il n'en demeure pas moins que nous connaissons plutôt mal l'histoire de la zone de la Faille de Cadillac où se trouvent concentrées la plupart des mines de la première génération et les principales agglomérations de la région. L'activité minière a pourtant été l'un des principaux facteurs de l'extension du domaine habité de l'Abitibi-Témiscamingue. Celle-ci tire son importance non seulement de la valeur de la production minérale, du nombre de personnes occupées dans le secteur minier ou des villes et des empires miniers qu'elle a fait naître, mais aussi du rôle qu'elle a joué dans la consolidation du domaine agricole et dans le développement des infrastructures de communication. Bien plus, la colonisation minière, en s'insérant entre le vieux Témiscamingue et l'Abitibi rural, a comblé le vide entre les deux parties de la région déjà aménagées.

Un certain nombre d'ouvrages, de thèses et d'études diverses furent tout de même consacrés, en tout ou en partie, à l'histoire des mines de l'Abitibi-Témiscamingue ${ }^{2}$. Il existe de nombreuses façons de rendre compte de cette documentation. Nous avons choisi, pour les fins de ce court aperçu critique, de classer ces différents documents en fonction du type d'informations et d'explications historiques fournies.

\section{L'OCCUPATION DU TERRITOIRE ET LE DÉVELOPPEMENT MINIER}

Les livres des géographes Raoul Blanchard, Pierre Biays et Marcien Villemure constituent, selon nous, les ouvrages de base ${ }^{3}$. Ces géographes sont les premiers, et les seuls jusqu'à présent, à avoir étudié de manière globale et cohérente l'occupation et le développement minier de l'Abitibi-Témiscamingue. Cette trilogie nous fournit une information abondante et de précieuses explications sur la géologie, les premières explorations, la mise en valeur et l'aménagement de la région minière.

2 Voir B.-B. Gourd, Bibliographie de l'Abitibi-Témiscamingue (Rouyn, Université du Québec, Direction des Études universitaires dans l'Ouest québécois, 1973), x-270p.; Bibliographie de l'Abitibi-Témiscamingue. Supplément (Rouyn, Université du Québec, Direction des Études universitaires dans l'Ouest québécois, 1975), ix214 p.

R. Blanchard, L'Ouest du Canada français. Province de Québec - 2 Les pays de l'Ottawa. L'Abitibi-Témiscamingue (2 vol., Montréal, Beauchemin, 1954), 325 p.; P. Biays, Les marges de l'ckoumène dans l'Est du Canada (Québec, Presses de l'Université Laval, 1964), 760 p.; M. Villemure, Les villes de la Faille de Cadillac (Rouyn, Conseil économique régional du Nord-Ouest québécois, 1971), 160 p. 
Les pays de l'Ottawa. L'Abitibi-Témiscamingue fait partie de la monumentale géographie du Québec de Raoul Blanchard. L'auteur ne consacre qu'un nombre limité de pages aux mines de la région, mais elles sont d'une grande densité. Soulevons cependant deux points faibles. Blanchard constate l'influence de l'Ontario dans la mise en valeur du domaine minier de la région. Il nous fournit toutefois peu d'explications sur son importance et sur ses causes. Il aurait dû d'autre part davantage situer dans le temps la colonisation agricole et le développement minier. Il nous semble en effet fondamental de comprendre que, dans le cas de l'Abitibi-Témiscamingue, les deux types d'occupation du territoire se chevauchent à partir de 1925 dans le temps et l'espace. Il faut savoir par exemple que le grand «rush» minier de Val d'Or se situe à la même époque que les plans de colonisation Gordon et Vautrin. Ceci dit, il faut s'empresser de souligner que le livre demeure encore, plus de vingt ans après sa publication, l'instrument indispensable à toute recherche sur l'histoire de l'Abitibi-Témiscamingue.

Les marges de l'ckoumène dans l'Est du Canada porte sur le problème des limites de l'occupation des territoires vierges de la partie orientale du Bouclier canadien. La seconde partie du livre, traitant des conquêtes humaines, reste sans contredit celle dont l'historien tire le plus grand profit. Biays y étudie les diverses formes d'exploitation des ressources qui ont contribué, de façon inégale dans l'espace et variable dans le temps, au peuplement des régions marginales de l'Est du Canada. Il innove au niveau de la colonisation minière en expliquant les conditions juridiques et politiques de l'expansion minière. Son étude permet également de faire d'utiles comparaisons entre le développement minier du Nord-Est ontarien et celui du Nord-Ouest québécois. Biays s'en remet toutefois entièrement à Blanchard pour la phase "héroïque" (1910-1950) de la colonisation minière de l'Abitibi-Témiscamingue. Cela surprend, surtout après le remaniement qu'il fait subir aux explications de Blanchard concernant le peuplement agricole. Biays s'est plutôt appliqué à décrire la phase difficile que traverse la zone minière depuis 1950 et à analyser les possibilités de reconversion économique des villes de la Faille de Cadillac.

Le livre de Marcien Villemure se penche pour sa part sur l'évolution de la Faille de Cadillac et les agglomérations qui s'y trouvent. L'ouvrage débute par une description géologique de la zone minéralisée et par l'étude du type de gisements en exploitation, du nombre et du genre de mines. La deuxième partie du livre porte sur l'urbani- 
sation de la zone, sa croissance démographique et l'évolution de ses différents secteurs économiques. Villemure termine par une réflexion sur l'avenir des villes minières. Son étude profite des travaux de Blanchard et de Biays pour analyser l'évolution de la région minière de ses origines jusqu'aux années soixante. Son recours aux statistiques régionales constitue son principal apport personnel. Villemure vient en effet étayer les études de ses prédécesseurs en nous donnant de nombreux tableaux détaillés sur les gisements, les mines, la démographie urbaine et la croissance économique. Il s'avoue cependant incapable de décrire de manière précise l'évolution des emplois dans le secteur minier à cause des lacunes des statistiques gouvernementales à ce chapitre.

Ces trois livres ne peuvent évidemment pas répondre à toutes les questions concernant l'histoire du développement minier de la région. Il faut également se documenter sur les ensembles dans lesquels s'insère l'Abitibi-Témiscamingue ou sur des aspects limités de ce développement mais particulièrement instructifs. La thèse de J. F. Gregory et les livres de E. S. Moore et de Leslie Roberts constituent les meilleurs exemples de ces travaux complémentaires ${ }^{4}$.

Les deux premiers nous expliquent l'appartenance de l'Abitibi-Témiscamingue au vaste ensemble géographique et économique que forme la zone Ontario-Québec du Bouclier canadien. Le développement minier de ce territoire s'effectue de manière continue de 1848 à 1950. Sa progression géographique, en s'étalant en sept districts miniers de Sudbury à Chibougamou, est nettement caractéristique ${ }^{5}$. La mise en exploitation des ressources minières de cette région centrale du Bouclier canadien s'opère sous l'impulsion des besoins de l'industrie américaine en métaux, avec des capitaux américains, à l'aide de procédés techniques mis au point aux États-Unis ou adaptés aux conditions canadiennes par des techniciens et ingénieurs américains. L'accumulation de capital et l'acquisition de connaissances techniques par des groupes ontariens à partir de Cobalt va toutefois permettre l'émergence d'une industrie minière canadienne.

4 J.F. Gregory, A Geographical Analysis of the Ontario-Quebec Metals Region (Philadelphie, thèse de M.Sc., Pennsylvania State University, 1959), 211 p.; E.S. Moore, American Influence in Canadian Mining (Toronto, University of Toronto Press, 1941), 144 p.; L. Roberts, Noranda (Toronto, Clarke \& Irwin, 1952), 233 p.

5 Ces districts sont ceux de Sudbury, Cobalt, Porcupine-Timmins, Larder Lake-Kirkland Lake, Rouyn, Malartic-Val d'Or et Chibougamou. 
Noranda, de Leslie Roberts, se penche pour sa part sur un point limité mais révélateur de l'histoire de la région minière. Le livre décrit l'édification de l'empire minier de Noranda Mines Limited $^{6}$. Il retrace l'itinéraire de la croissance de l'entreprise minière qui conduit des premières explorations de Horne et du «Lake Tremoy Syndicate», dans la région de Rouyn, jusqu'à l'existence de la multinationale canadienne du début des années 1950. Noranda, écrit à la demande de la société minière, s'applique principalement à faire ressortir la réussite de Noranda Mines Limited. L'ouvrage nous apprend ainsi beaucoup sur l'entreprise, mais bien peu sur RouynNoranda et la région minière.

\section{LE VÉCU}

Ces différents ouvrages et ces études forment la documentation de base. Elle demeure malgré tout incomplète. La dimension humaine du développement minier de l'Abitibi-Témiscamingue reste absente de ces ouvrages, sauf peut-être chez Blanchard et Roberts. Il faut donc recourir à une autre catégorie de documents, ceux qui témoignent du vécu.

\section{L'ÉPOPÉE MINIÈRE}

Un grand nombre d'ouvrages apologétiques ont été consacrés aux grandes découvertes minières canadiennes de la fin du XIX siècle et de la première moitié du $\mathrm{XX}^{\mathrm{e}}$ siècle. L'AbitibiTémiscamingue participe à cette grande épopée minière dans plusieurs de ces livres qui réservent à la région quelques pages ou quelques chapitres ${ }^{7}$. Ces ouvrages ne distinguent pas les diverses phases

6 Sur l'histoire de Noranda Mines Limited et de la mine Horne, on peut aussi lire W.A. Buik, Noranda Mines Limited: A Study in Business and Economic History (Toronto, thèse de M.A. présentée à l'Université de Toronto, 1958), 21022p.; G. Fournier, Historique de la mine Noranda (Montréal, thèse de B.Sc. présentée à l'École polytechnique, 1962), 34 p.

Signalons les principaux; A.D. Hoffman, Free Gold. The Story of Canadian Mining (New-York, Rinehart \& Co., 1947), 420 p.; D.M. Lebourdais, Metals and Men. The Story of Canadian Mining (Toronto, McClelland \& Stewart, 1957), 416 p.; B.F. Townsley, Mine-Finders. The History and Romance of Canadian Minerals Discoveries (Toronto, Saturday Night Press, 1935), 246 p.; J.B. MacDougall, Two Thousand Miles of Gold. From Val d'Or to Yellowknife (Toronto, McClelland \& Stewart, 1946), 234 p.; L.F. Jones, G. Lonn et R.M. Longo, Historical Highlights of Canadian Mining (Toronto, Pitts Publishing Co., 1973), 274 p.; G. Lonn, The Mine Finders (Toronto, Pitts Publishing Co., 1966), 237 p.; G. Lonn, Builders of Fortunes, (Toronto, Pitts Publishing Co., 1963), 118 p. 
de l'expansion minière, ne se préoccupent guère d'en cerner les différents aspects, et font habituellement peu de rapprochements entre le développement minier et la conjoncture économique et politique du Canada du temps. D'ailleurs, là ne réside pas leur propos. Ils s'appliquent plutôt à raconter «l'aventure» des bâtisseurs de l'industrie minière canadienne. Il va sans dire qu'ils sont plus enclins à louanger qu'à faire comprendre et que plusieurs d'entre eux possèdent un côté anecdotique très marqué. Ils fourmillent pourtant de mille et un détails qui viennent rendre vie aux études scientifiques. Ils dépeignent ainsi assez bien la confrérie aux multiples ramifications que formaient les pionniers de l'industrie minière. Ces livres apparaissent également révélateurs de l'envoûtement exercé à l'époque sur de larges secteurs de l'opinion canadienne par les découvertes minières. Ces ouvrages, parce qu'ils racontent l'histoire des mines dans une optique pancanadienne, nous apprennent très peu de choses sur l'Abitibi-Témiscamingue minier.

L'Abitibi, pays de l'or, d'Émile Benoist, nous renseigne par contre beaucoup mieux sur la vie de la région minière de la période 1910-1950 ${ }^{8}$. Ce livre, qui regroupe les articles que le journaliste publia dans Le Devoir, reconstitue l'ambiance de la région minière de 1937, particulièrement celle de Val d'Or et des camps miniers de Malartic et Cadillac. Son originalité réside dans le fait qu'il aborde des questions qui ont échappé aux observateurs canadiens, ou qui, plus simplement, ne leur semblaient pas très significatives. Benoist remarque par exemple que la domination des entreprises minières sur la vie socio-économique de villes comme Val d'Or se prolonge profondément dans la vie culturelle. L'auteur souligne, comme d'autres observateurs de l'époque, la confusion et l'absence totale de planification qui ont présidé à la naissance des villes minières. Il reste toutefois un des rares à décrire le dédoublement administratif des principales agglomérations minières. Blanchard s'en inspira sans doute pour faire sa comparaison entre Westmount-Montréal et Noranda-Rouyn, Bourlamaque-Val d'Or. Benoist constate également que, déjà en 1937, l'activité minière a amené le déplacement des centres d'influence de la région. Amos, capitale de l'Abitibi des

8 E. Benoist, L'Abitibi, pays de l'or (Montréal, Éditions du Zodiaque, 1938), 198 p. Consulter également D. Potvin, Sous le signe du quartz. Histoire romancée des mines du Nord-Ouest québécois (Montréal, Éditions Bernard Valiquette, 1940), 262 p.; A. Pelletier, J'ai vu naître et grandir ces jumelles (Rouyn, Imprimerie Lebonfon, 1967), 194 p.; R. Jodouin, En-d'ssour (Montréal, Éditions québécoises, 1973), 207 p. 
premières années de colonisation agricole, cède sa place aux villes de Rouyn et de Val d'Or. Ce sont là évidemment les observations d'un journaliste en tournée. Les incohérences et les répétitions sont nombreuses. Le style journalistique du livre lui fait toutefois gagner en spontanéité et en fraîcheur ce qu'il peut perdre en rigueur. Benoist nous donne ainsi plusieurs pages vivantes sur les «Paris-laNuit» et les autres villages clandestins, situés à la périphérie des villes minières, qui peuplèrent le paysage de la région durant les belles années de la ruée vers l'or.

\section{Les travailleurs miniers}

Tous ces livres consacrés à l'épopée minière de l'AbitibiTémiscamingue ne recréent pourtant qu'une partie de la vie de la région. Les mines et la région minière, par on ne sait quel curieux hasard, auraient été uniquement bâties par une poignée de prospecteurs et d'entrepreneurs audacieux. Les ouvrages passent en effet totalement sous silence les conditions de travail et d'existence des travailleurs miniers. L'histoire de ces ouvriers reste encore méconnue. Un chapitre du livre d'Evelyn Dumas, Dans le sommeil de nos os, et une thèse sur les mineurs immigrants à Rouyn-Noranda constituent les principales pièces à verser au dossier de l'histoire des travailleurs miniers de la période $1910-1950^{9}$.

Evelyn Dumas reconstitue dans son livre la trame historique de 12 grèves majeures du Québec de la période 1934-1944 en se servant abondamment des témoignages des contemporains. Un chapitre, celui sur la grève des mineurs de la Noranda en 1934, nous intéresse particulièrement, et ce à plus d'un titre. Ce cas nous paraît caractéristique des grèves de courte durée, mais d'une grande âpreté, menées par des syndicats radicaux dans les régions éloignées et isolées du Nord du Québec et du Canada. Ces conflits eurent à l'époque très peu d'échos dans les grands centres et leur souvenir se serait

9 E. Dumas, Dans le sommeil de nos os. Quelques grèves au Québec de 1934 à 1944 (Montréal, Leméac, 1971), 170 p.; F. Larouche, L’immigrant dans une ville minière du Québec (thèse de M.A. présentée à l'Université Laval, 1974), 91 p. ; un résumé de cette thèse a été publiée dans Recherches sociographiques, vol. 14, no 2 (1973): 203-228 et dans B.-B. Gourd, dir., Abitibi-Témiscamingue. Quatre études sur le Nord-Ouest québécois (Rouyn, Presses du CEGEP de Rouyn-Noranda, 1974), 26-63. Consulter aussi F. Côté, 'L'industrie minière et le problème social', Actualité économique, vol. 21, t. 2, no 5 (1946): 409-427; et pour la période postérieure à 1950, J. Mehling, Analyse socio-économique d'une grève (Montréal, Presses de l'École des Hautes Études commerciales et Beauchemin, 1963), 218 p. 
perdu dans la nuit des temps sans des travaux comme ceux d'Evelyn Dumas. Le récit de ce conflit nous intéresse également parce qu'il porte sur la première grève touchant l'industrie minière de l'Abitibi-Témiscamingue. Il est riche en renseignements sur ce que pouvaient être les conditions des mineurs et les relations de travail dans les mines de la région durant les années 1930. L'étude, par contre, nous donne assez peu d'informations sur le climat social régnant dans la région de Rouyn. Le district minier connaît en effet, de 1929 à 1935, une vie sociale extrêmement agitée et les mouvements politiques et ouvriers radicaux sont solidement implantés dans le milieu des mineurs d'origine étrangère. Evelyn Dumas, en analysant 12 grèves en 170 pages, ne fait évidemment qu'un premier survol de l'histoire des luttes ouvrières du temps.

L'immigrant dans une ville minière au Québec, de Fernand Larouche, nous aide aussi à mieux comprendre l'histoire des mineurs. À partir du cas de Rouyn-Noranda, le sociologue étudie au niveau du vécu comment les immigrants perçoivent le réseau de relations existant entre les groupes francophone et anglophone, et comment ils cherchent à s'y intégrer. Précisons ici que l'étude porte sur les mineurs immigrants arrivés dans la région durant les années 19451950 et recrutés en Europe parmi les personnes déplacées par la guerre. Fernand Larouche constate que chaque groupe est demeuré à la périphérie du cercle des valeurs significatives communes aux autres groupes. Ces relations demeurent de plus très souvent ambivalentes. Les immigrants ont préféré s'assimiler à la culture du groupe anglophone dominant parce qu'elle présentait au niveau de l'interaction beaucoup plus de constance et de stabilité, et qu'elle pouvait s'appuyer sur des réalisations concrètes comme l'entreprise minière. L'auteur éprouve cependant certaines difficultés à insérer son analyse sociologique dans la réalité historique du développement minier. Il n'a pas cherché d'autre part à savoir ce que pensaient les différents groupes de mineurs du syndicalisme et des rapports entre les ouvriers et les compagnies minières.

Ces deux études ne portent que sur des aspects limités de l'histoire des travailleurs miniers. Il faut nécessairement replacer celle-ci dans le contexte plus global du syndicalisme minier et des luttes ouvrières du Québec et du Canada. La consultation de travaux comme ceux d'Irving Abella et de John Lang devient dès lors très utile ${ }^{10}$.

10 I. Abella, Nationalism, Communism and Canadian Labour: The C.I.O., the Communist Party and the Canadian Congress of Labour 1935-1956 (Toronto, 


\section{CONCLUSION}

Cet aperçu de la littérature pouvant servir à l'histoire du développement minier de la période 1910-1950 est forcément incomplet. Il ne se préoccupe que des études qui nous paraissent les plus importantes. Il laisse ainsi de côté les ouvrages portant sur la géologie de la région. La Commission géologique du Canada et le Ministère des Mines du Québec ont publié plusieurs centaines de volumes. Un grand nombre d'entre eux possèdent une valeur historique certaine $^{11}$. Il en va de même de revues spécialisées telles que Canadian Mining Journal ou Canadian Mining and Metallurgical Bulletin ${ }^{12}$. Nous n'avons pas fait mention également d'études générales qui, comme celles de J.L. Robinson ou M. Zaslow, contiennent de nombreux éléments explicatifs pertinents à l'Abitibi-Témiscamingue ${ }^{13}$.

Cet examen nous permet malgré tout d'évaluer la documentation sur laquelle l'historien peut compter au départ. On prend aussi rapidement conscience du caractère embryonnaire de l'histoire des mines de la région. De multiples aspects de cette histoire demeurent encore dans l'ombre. Plusieurs questions fondamentales restent toujours sans réponse. Les travaux mentionnés nous indiquent par contre de nombreuses pistes de recherche et sont riches en hypothèses de travail. L'intérêt nouveau pour l'histoire régionale et le développement des études collégiales et universitaires en AbitibiTémiscamingue laissent espérer que ce travail vers une meilleure connaissance du milieu se poursuivra ${ }^{14}$.

University of Toronto Press, 1973), 256 p.; J. Lang, A Lion in a Den of Daniels. A History of the International Union of Mine, Mill and Smelter Workers in Sudbury, Ontario 1942-1962 (Guelph, thèse de M.A. présentée à l'Université de Guelph, 1970), $335 \mathrm{p}$.

11 Voir principalement Rapport sur les opérations minières de la Province de Québec (1904-1928); Rapport annuel du Service des mines de Québec (1929-1936); L'industrie minière de la Province de Québec (1937).

12 Canadian Mining Journal a ainsi consacré durant la période étudiée trois numéros complets à la région: vol. 55, no 4 (avril 1934); vol 57, no 10 (octobre 1936); vol. 59, no 8 (août 1938).

13 J.L. Robinson, Resources of the Canadian Shield (Toronto, Methuen Publications, 1969), 136 p.; M. Zaslow, The Opening of Canadian North 1870-1914 (Toronto, McClelland \& Stewart, 1971), 339 p.

14 À ce propos on peut consulter les deux premiers numéros des «Cahiers du Département d'histoire et de géographie»publiés en 1974 et 1975 par le Collège du Nord-Ouest. 